\title{
Short-term outcomes and nutritional status after laparoscopic subtotal gastrectomy with a very small remnant stomach for cStage I proximal gastric carcinoma
}

\author{
Haruna Furukawa $^{1} \cdot$ Yukinori Kurokawa $^{1} \cdot$ Shuji Takiguchi $^{1} \cdot$ Koji Tanaka $^{1}$. \\ Yasuhiro Miyazaki $^{1} \cdot$ Tomoki Makino $^{1} \cdot$ Tsuyoshi Takahashi $^{1} \cdot$ Makoto Yamasaki $^{1}$. \\ Kiyokazu Nakajima ${ }^{1} \cdot$ Masaki Mori $^{1} \cdot$ Yuichiro Doki $^{1}$
}

Received: 26 May 2017 / Accepted: 28 July 2017 / Published online: 20 August 2017

(C) The International Gastric Cancer Association and The Japanese Gastric Cancer Association 2017

\begin{abstract}
Background Total or proximal gastrectomy is usually performed for early proximal gastric carcinoma, but the optimal type of gastrectomy is still unknown. We evaluated shortterm outcomes and nutritional status after laparoscopic subtotal gastrectomy (LsTG) in comparison with laparoscopic total gastrectomy (LTG) and laparoscopic proximal gastrectomy (LPG).

Methods We analyzed 113 patients who underwent LsTG $(n=38)$, LTG $(n=48)$, or LPG $(n=27)$ for cStage I gastric cancer located in the upper third of the stomach. Postoperative morbidities, nutritional status including body weight, serum albumin, hemoglobin, the prognostic nutritional index (PNI), and endoscopic findings at 1 year after surgery were compared between LsTG and both LTG and LPG.

Results Operation time and intraoperative blood loss were similar among the three groups. The incidence of postoperative morbidities was lower in LsTG than in LTG. The degree of body weight loss was significantly smaller in LsTG than in LTG at 6 and 12 months. At 12 months, LsTG resulted in better serum albumin and PNI than LPG, and better hemoglobin than LTG. Endoscopic examination demonstrated that one LsTG patient and two LPG patients had reflux esophagitis. Remnant gastritis was observed more frequently in LPG than in LsTG. No LsTG patient had bile reflux, although it was observed in four LPG patients.

Conclusions LsTG with a very small remnant stomach had favorable short-term outcomes and nutritional status
\end{abstract}

Yukinori Kurokawa

ykurokawa@gesurg.med.osaka-u.ac.jp

1 Department of Gastroenterological Surgery, Graduate School of Medicine, Osaka University, 2-2-E2, Yamadaoka, Suita, Osaka 565-0871, Japan compared with LTG and LPG, so it may be a better treatment option for cStage I proximal gastric carcinoma.

Keywords Early gastric cancer - Proximal gastric carcinoma $\cdot$ Laparoscopic subtotal gastrectomy $\cdot$ Small remnant stomach

\section{Background}

Gastric cancer is still a common malignancy worldwide, and its incidence in the upper third of the stomach has been increasing [1-3]. Early gastric cancer in the upper third of the stomach is usually treated with total or proximal gastrectomy. Total gastrectomy (TG) provides more complete lymphadenectomy, has no risk of remnant gastric cancer, and is associated with a lower incidence of reflux esophagitis than proximal gastrectomy (PG) [4-6]. However, TG has many disadvantages from the nutritional aspect, such as postoperative body weight loss and anemia [7]. On the other hand, PG has several benefits compared to TG in terms of maintaining gastric hormones and gastric acid production resulting from preservation of the gastric antrum. However, reflux esophagitis is common after PG, and although various reconstruction methods have been suggested to reduce this problem, no standard procedure has yet been established [5, 7-11].

A recently reported procedure, laparoscopic subtotal gastrectomy (LsTG) with a very small remnant stomach, has demonstrated advantages in postoperative morbidities and nutritional status compared with laparoscopic TG (LTG) $[12,13]$. One of the most important roles of LsTG is to preserve ghrelin, which is mainly produced in the fundic gland of the stomach [14]. Therefore, the preservation of the fundus but not the antrum may preserve nutritional status after 
gastrectomy. However, no studies have compared LsTG with laparoscopic PG (LPG), so there is still no consensus about whether LsTG is a viable treatment option for gastric cancer located in the upper third of the stomach. In the present study, we evaluated the short-term outcomes and nutritional status after LsTG in comparison with LTG and LPG.

\section{Patients and methods}

\section{Patients}

Eligible patients were those who underwent LsTG, LTG, or LPG for clinically diagnosed stage I gastric cancer located in the upper third of the stomach at Osaka University Hospital between January 2010 and July 2016. Clinical stage was classified according to the Japanese Classification of Gastric Carcinoma [15]. Exclusion criteria were as follows: double cancer involving the stomach and another organ, esophagogastric junctional cancer, conversion laparotomy, and emergency surgery cases. Among the eligible 113 patients, LsTG, LTG, and LPG were performed for 38, 48, and 27 patients, respectively. All patients received either D1+ or D2 lymphadenectomy according to the Japanese Gastric Cancer Treatment Guidelines [16]. In principle, patients with pStage II/III underwent adjuvant S-1 therapy ( $80-120 \mathrm{mg} /$ body) for 1 year. This study was approved by the institutional review board of the Osaka University Hospital.

\section{Surgical procedure}

The surgical procedure (LsTG, LTG, or LPG) was determined based on tumor location and the patient's wishes. When a tumor was located within $2 \mathrm{~cm}$ of the esophagogastric junction (EGJ), LsTG was contraindicated, and LPG was contraindicated for tumors spreading to the gastric angle.
In this study, LsTG was defined, according to the previous study, as follows: (1) laparoscopic distal gastrectomy with Roux-en-Y reconstruction; (2) tumors located in or spreading to the upper third of the stomach; (3) a certain distance from the tumor to the EGJ remained but the distance was $<5 \mathrm{~cm}$; and (4) the remnant gastric stump was 2-3 cm from the EGJ [12]. Almost all LsTG cases required total resection of the lesser curvature of the stomach. However, at least one of the short gastric vessels near the cardia was preserved as blood supply to the remnant stomach. The resection line of the stomach in the LsTG procedure varied according to the location of the tumor (Fig. 1). We usually put the marking clips approximately $1 \mathrm{~cm}$ proximal to the tumor with confirmation of negative biopsy. In addition, the tumor location and marking clips were confirmed by using intraoperative endoscopy before resection, and intraoperative frozen section diagnosis was performed in some cases. A gastrojejunal antecolic anastomosis was created using a linear stapler or a $25-\mathrm{mm}$ circular stapler. A side-to-side jejunojejunostomy was performed $30 \mathrm{~cm}$ distal from the gastrojejunal anastomosis.

In the LTG procedure, the antecolic esophagojejunostomy was performed using a $25-\mathrm{mm}$ circular stapler for the purse-string suture method or a linear stapler for the overlap method, as previously reported [17]. A side-to-side jejunojejunostomy was then performed $45 \mathrm{~cm}$ distal from the esophagojejunal anastomosis.

In the LPG procedure, esophagogastric anastomosis or double-tract reconstruction was performed. An esophagogastric anastomosis was performed by anastomosing the abdominal esophagus with the anterior wall of the remnant stomach using a $25-\mathrm{mm}$ circular stapler. For esophagogastric anastomosis cases, Toupet-like partial fundoplication was performed according to a previous report [18]. For doubletract reconstruction cases, esophagojejunostomy was performed using a $25-\mathrm{mm}$ circular stapler or linear stapler as in the LTG procedure. A jejunogastrostomy was performed
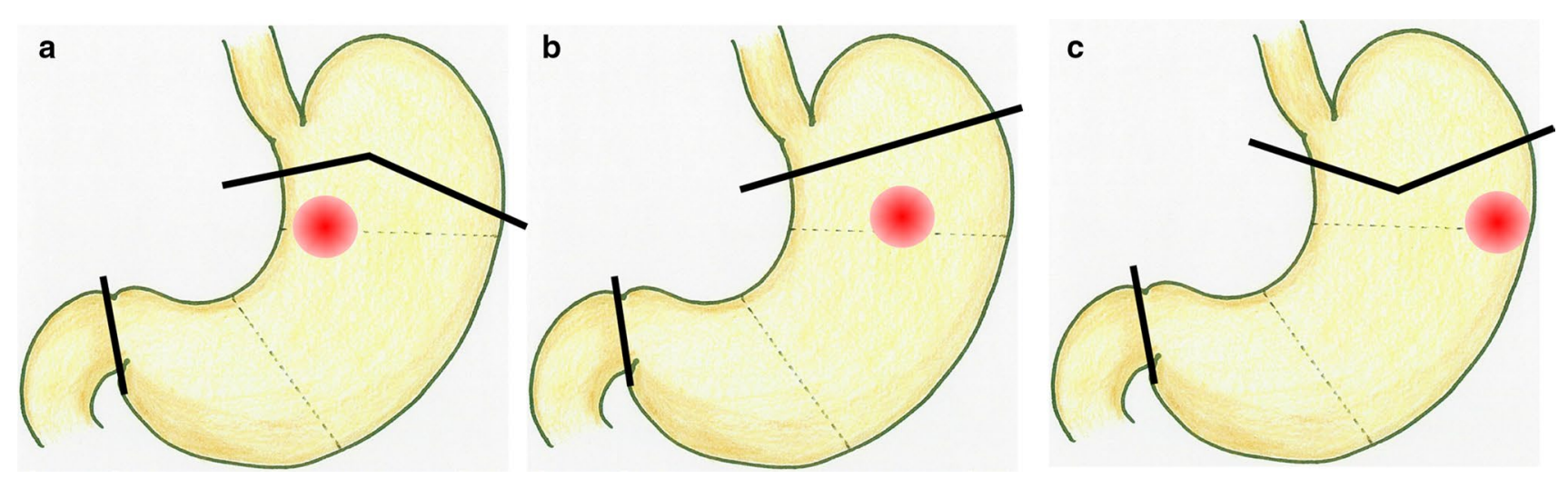

Fig. 1 Variation in resection lines in laparoscopic subtotal gastrectomy (LsTG) for tumors located in the lesser curvature (a), anterior or posterior wall (b), and greater curvature (c) 
using a linear stapler, with interposition of $15-20 \mathrm{~cm}$ of jejunum between the esophagojejunostomy and jejunogastrostomy. A side-to-side jejunojejunostomy was performed $20 \mathrm{~cm}$ distal from the jejunogastric anastomosis.

\section{Outcomes}

The short-term outcomes evaluated in this study were operation time, blood loss, and postoperative morbidities within 30 days after surgery. Postoperative morbidities were classified according to the Clavien-Dindo classification of surgical complications, and those of grade II or higher were recorded $[19,20]$. Nutritional parameters after gastrectomy were assessed on the basis of changes in body weight, serum albumin, hemoglobin, and the prognostic nutritional index (PNI) at 1, 6, and 12 months after surgery. PNI was calculated using the following formula: $10 \times$ serum albumin value $(\mathrm{g} / \mathrm{dl})+0.005 \times$ lymphocyte count in peripheral blood. Endoscopic examinations were performed 1 year after surgery for patients who underwent LsTG or LPG to evaluate reflux esophagitis, gastritis, bile reflux, and the presence of residual food in the remnant stomach according to the RGB (residue, gastritis, bile) classification [21].

\section{Statistical analysis}

Statistical differences between the LsTG and the LTG/LPG groups were calculated with the chi-square test and Fisher's exact test for categorical variables and with the Mann-Whitney $U$ test for continuous variables. Postoperative changes in body weight and nutritional parameters were compared between the two groups by Student's $t$ test. Statistical significance was defined as $P<0.05$. The statistical analysis was performed using the JMP (version 11.0) software program (SAS Institute, Cary, NC, USA).

\section{Results}

\section{Patient characteristics}

The clinicopathological characteristics of patients who underwent LsTG, LTG, or LPG are detailed in Table 1. There were no significant differences in patient background between the LsTG and the LTG and LPG groups except for lymph node dissection (LsTG vs. LPG) and the type of stapler used for anastomosis (LsTG vs. both LTG and LPG). Thirteen patients in the LsTG group and 16 in the LTG group underwent D2 lymphadenectomy, and all patients in the LPG group underwent D1+ lymphadenectomy. Clinical and pathological stages were similar among all groups. Although there was no significant difference in the proportion of patients who received adjuvant chemotherapy, slightly more patients underwent adjuvant chemotherapy in the LsTG group than in the LPG group (16\% vs. $4 \%$ ). In the LPG group, 21 patients (78\%) underwent double-tract

Table 1 Patient characteristics

\begin{tabular}{|c|c|c|c|c|c|}
\hline & LsTG $(n=38)$ & LTG $(n=48)$ & $\begin{array}{l}P \text { value for } \\
\text { LsTG vs. LTG }\end{array}$ & LPG $(n=27)$ & $\begin{array}{l}P \text { value for } \\
\text { LsTG vs. } \\
\text { LPG }\end{array}$ \\
\hline Age, median (range) & $65(44-86)$ & $63.5(29-82)$ & 0.209 & $70(59-84)$ & 0.124 \\
\hline Sex, male/female & $26 / 12$ & $35 / 13$ & 0.811 & $22 / 5$ & 0.268 \\
\hline BMI $\left(\mathrm{kg} / \mathrm{m}^{2}\right)$, median (range) & $22.6(16.6-32.5)$ & $22.2(13.9-26.5)$ & 0.972 & $22.8(19.3-26.8)$ & 0.299 \\
\hline ASA-PS, $1 / 2 / 3$ & $18 / 18 / 2$ & $30 / 16 / 2$ & 0.371 & $8 / 18 / 1$ & 0.303 \\
\hline Tumor location, Ant/Post/Gre/Less & $5 / 10 / 5 / 18$ & $10 / 12 / 5 / 21$ & 0.818 & $2 / 8 / 1 / 16$ & 0.475 \\
\hline Tumor size (mm), median (range) & $30(10-70)$ & $32(10-100)$ & 0.309 & $25(12-50)$ & 0.305 \\
\hline Histological type, differentiated/undifferentiated & $19 / 19$ & $24 / 24$ & 1.000 & $15 / 12$ & 0.802 \\
\hline $\mathrm{cT}, \mathrm{T} 1 / \mathrm{T} 2$ & $33 / 5$ & $40 / 8$ & 0.767 & $26 / 1$ & 0.388 \\
\hline $\mathrm{cN}, \mathrm{N} 0 / \mathrm{N} 1$ & $37 / 1$ & $48 / 0$ & 0.432 & $27 / 0$ & 1.000 \\
\hline cStage, IA/IB & $32 / 6$ & $40 / 8$ & 1.000 & $26 / 1$ & 0.224 \\
\hline Lymph node dissection, D1+/D2 & $25 / 13$ & $32 / 16$ & 1.000 & $27 / 0$ & $<0.001$ \\
\hline Stapler used for anastomosis, circular/linear & $18 / 20$ & $38 / 10$ & 0.003 & $23 / 4$ & 0.002 \\
\hline $\mathrm{pT}, \mathrm{T} 1 / \mathrm{T} 2 / \mathrm{T} 3-4$ & $31 / 3 / 4$ & $32 / 9 / 7$ & 0.258 & $24 / 0 / 3$ & 0.327 \\
\hline $\mathrm{pN}, \mathrm{N} 0 / \mathrm{N} 1 / \mathrm{N} 2-3$ & $33 / 3 / 2$ & $41 / 3 / 4$ & 0.830 & $26 / 0 / 1$ & 0.306 \\
\hline pStage, I/II/III & $31 / 6 / 1$ & $39 / 4 / 5$ & 0.239 & $24 / 2 / 1$ & 0.589 \\
\hline Adjuvant chemotherapy, yes/no & $6 / 32$ & $6 / 42$ & 0.758 & $1 / 26$ & 0.224 \\
\hline
\end{tabular}

$L s T G$ laparoscopic subtotal gastrectomy, $L T G$ laparoscopic total gastrectomy, $L P G$ laparoscopic proximal gastrectomy, $B M I$ body mass index, ASA-PS American Society of Anesthesiologists physical status, Ant anterior wall, Post posterior wall, Gre greater curvature, Less lesser curvature 
reconstruction and only 6 patients (22\%) underwent esophagogastric anastomosis.

\section{Postoperative morbidity}

Surgical results and postoperative morbidities are shown in Table 2. Neither operation time nor intraoperative blood loss was significantly different between the LsTG group and the LTG and LPG groups. Regarding postoperative morbidities within 30 days after surgery, the overall incidence was lower in the LsTG group than in the LTG group (11\% vs. $31 \%, P=0.035)$. The incidences of anastomotic leakage and intraabdominal abscess were higher in the LTG group, but not significantly so. On the other hand, there were no significant differences in postoperative morbidities between the LsTG and LPG groups ( $11 \%$ vs. $19 \%, P=0.472$ ). There were no deaths consequent to surgery in all groups.

\section{Postoperative nutritional status}

We evaluated postoperative changes in body weight and nutritional parameters in 102 patients who were followed up for at least 1 year after surgery (Fig. 2). The degree of body weight loss was significantly smaller in the LsTG group than in the LTG group at both 6 months $(P<0.001)$ and 12 months $(P<0.001)$. On the other hand, there was a smaller decrease of body weight in the LsTG group as compared with the LPG group, although at no time point was it statistically significant. The serum albumin level recovered to the preoperative value at 12 months after surgery in the LsTG group but not in the LTG and LPG groups. In particular, the decrease of serum albumin in the LPG group at 12 months after surgery was significantly higher than that in the LsTG group $(P=0.020)$. The decrease in serum hemoglobin was greater in the LTG group throughout the year than in the other two groups, but not significantly so, and the LTG group showed a significantly higher decrease of hemoglobin at 12 months after surgery than the LsTG group $(P=0.043)$. Regarding the PNI, there was no significant difference between the LsTG and LTG groups; however, the decrease of PNI was significantly greater in the LPG group than in the LsTG group at 12 months after surgery $(P=0.007)$.

\section{Endoscopic findings}

Patients who underwent LsTG or LPG were required to undergo endoscopic examination during the follow-up period. At 1 year after surgery, 27 of 31 patients $(87 \%)$ in the LsTG group and 23 of 25 patients (92\%) in the LPG group underwent endoscopic examination. The endoscopic findings are shown in Table 3 . The endoscope could not reach the remnant stomach in 4 of 19 patients (21\%) who underwent LPG with double-tract reconstruction. One patient (4\%) in the LsTG group and 2 patients (9\%) in the LPG group were confirmed to have reflux esophagitis. Remnant gastritis was observed more frequently in the LPG group than in the LsTG group (37\% vs. $7 \%, P=0.022)$. No patient in the LsTG group exhibited bile reflux, which was observed in 4 LPG patients $(21 \%$, $P=0.024)$.

Table 2 Surgical results and postoperative morbidities

\begin{tabular}{|c|c|c|c|c|c|}
\hline & LsTG $(n=38)$ & LTG $(n=48)$ & $\begin{array}{l}P \text { value for LsTG } \\
\text { vs. LTG }\end{array}$ & LPG $(n=27)$ & $\begin{array}{l}P \text { value for } \\
\text { LsTG vs. } \\
\text { LPG }\end{array}$ \\
\hline Operation time (min), median (range) & $274(182-437)$ & $256.5(176-332)$ & 0.122 & $286(193-365)$ & 0.837 \\
\hline Blood loss (ml) median (range) & $75(0-370)$ & $60(10-400)$ & 0.973 & $90(0-580)$ & 0.215 \\
\hline Postoperative morbidity & $4(11 \%)$ & $15(31 \%)$ & 0.035 & $5(19 \%)^{\mathrm{a}}$ & 0.472 \\
\hline Anastomotic leakage & $1(3 \%)$ & $3(6 \%)$ & 0.627 & $3(11 \%)$ & 0.299 \\
\hline Anastomotic stricture & $0(0 \%)$ & $1(2 \%)$ & 1.000 & $0(0 \%)$ & - \\
\hline Bleeding & $1(3 \%)$ & $2(4 \%)$ & 1.000 & $1(4 \%)$ & 1.000 \\
\hline Pancreatic fistula & $0(0 \%)$ & $2(4 \%)$ & 0.501 & $1(4 \%)$ & 0.415 \\
\hline Delayed gastric emptying & $2(5 \%)$ & $0(0 \%)$ & 0.192 & $1(4 \%)$ & 1.000 \\
\hline Bowel obstruction & $0(0 \%)$ & $1(2 \%)$ & 1.000 & $0(0 \%)$ & - \\
\hline Intraabdominal abscess & $0(0 \%)$ & $4(8 \%)$ & 0.126 & $1(4 \%)$ & 0.415 \\
\hline Wound infection & $0(0 \%)$ & $1(2 \%)$ & 1.000 & $0(0 \%)$ & - \\
\hline Pneumoniae & $0(0 \%)$ & $1(2 \%)$ & 1.000 & $1(4 \%)$ & 0.415 \\
\hline Mortality, $n(\%)$ & $0(0 \%)$ & $0(0 \%)$ & - & $0(0 \%)$ & - \\
\hline
\end{tabular}

$L s T G$ laparoscopic subtotal gastrectomy, $L T G$ laparoscopic total gastrectomy, $L P G$ laparoscopic proximal gastrectomy

${ }^{\text {a }}$ Data include duplicate cases 

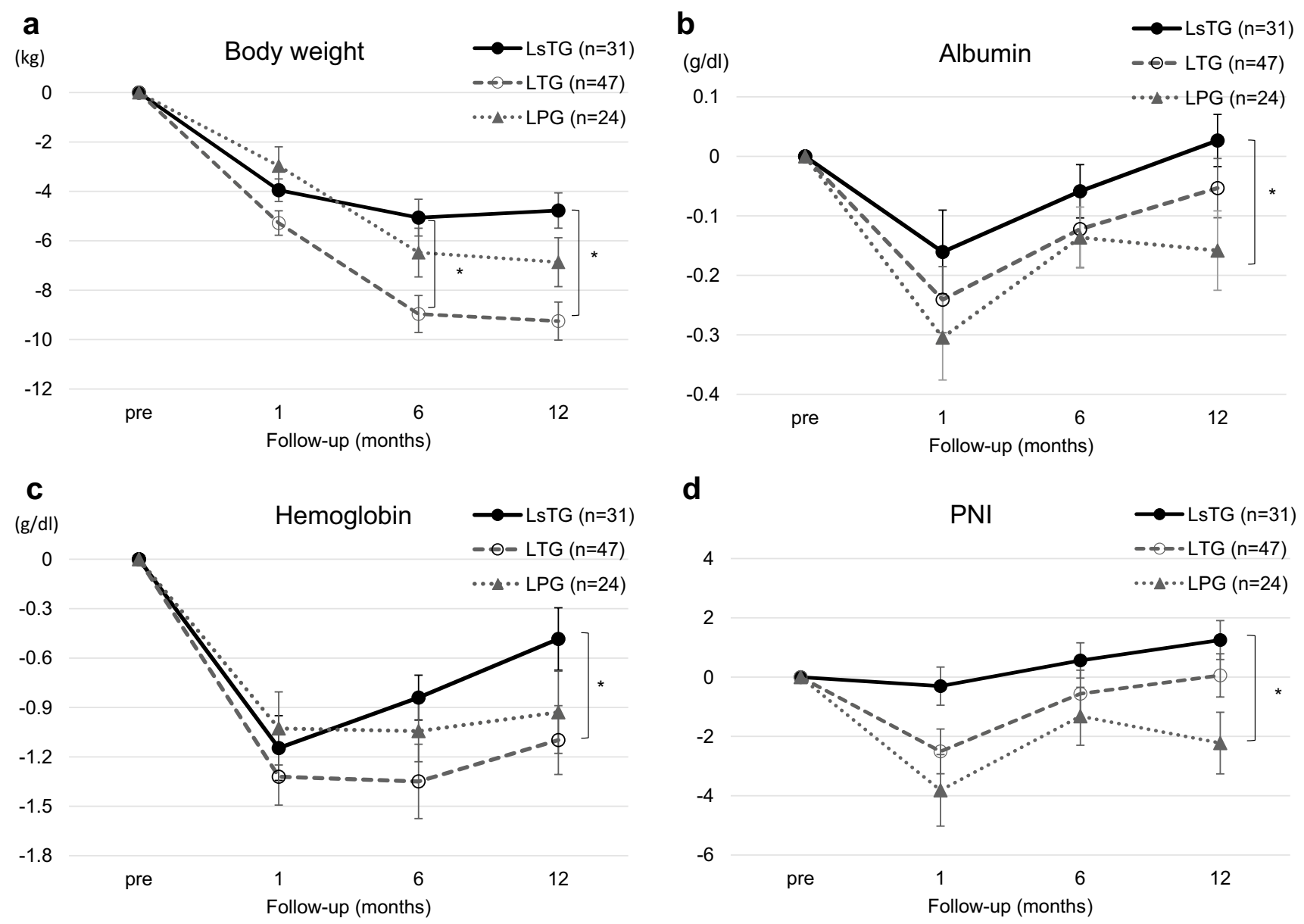

d

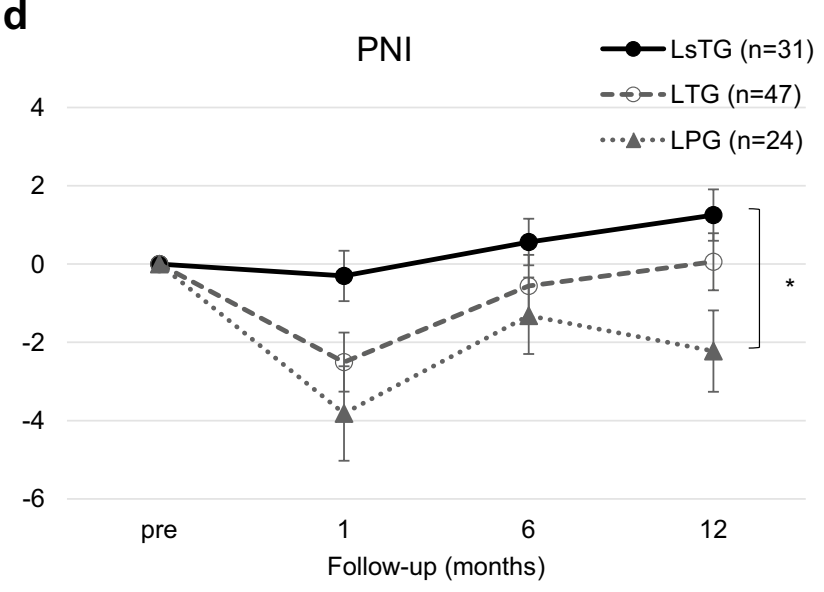

Fig. 2 Postoperative changes of body weight (a), serum albumin (b), serum hemoglobin (c), and prognostic nutritional index (PNI) (d) in the laparoscopic subtotal gastrectomy (LsTG) group, the laparoscopic

Table 3 Endoscopic findings at 1 year after surgery

\begin{tabular}{llll}
\hline & LsTG $(n=27)$ & LPG $(n=23)$ & $P$ value \\
\hline $\begin{array}{l}\text { Reflux esophagitis } \\
\text { RGB classification }\end{array}$ & $1(4 \%)$ & $2(9 \%)$ & 0.588 \\
$\quad \begin{array}{l}\text { Residual foods (grade } \\
\quad 0-1 / 2-4)\end{array}$ & $20 / 7$ & $15 / 4$ & 1.000 \\
$\quad \begin{array}{l}\text { Gastritis (grade 0-1/2-4) } \\
\text { Bile reflux (grade 0/1) }\end{array}$ & $25 / 2$ & $12 / 7$ & 0.022 \\
\hline
\end{tabular}

$L s T G$ laparoscopic subtotal gastrectomy, $L P G$ laparoscopic proximal gastrectomy, $R G B$ classification: residue, gastritis, bile classification

\section{Oncological outcomes}

The median follow-up periods for patients in the LsTG, LTG, and LPG groups were 31, 48.5, and 30 months, respectively. There was no recurrence in either the LsTG or LPG group, although two patients with pT3 gastric cancer in the LTG group developed recurrences and died of peritoneal

total gastrectomy (LTG) group, and the laparoscopic proximal gastrectomy (LPG) group. All postoperative data are represented as values (mean \pm standard error) relative to preoperative data. $* P<0.05$

metastasis. The 2-year recurrence-free survival rates were $100 \%$ in the LsTG group, $96 \%$ in the LTG group, and 100\% in the LPG group.

\section{Discussion}

In the present study, we assessed the short-term outcomes and nutritional status of patients who underwent LsTG with a very small remnant stomach for cStage I proximal gastric carcinoma, and compared these results with patients who underwent LTG and LPG. The morbidity rate was similar between the LsTG and LPG groups but was significantly higher in the LTG group than in the LsTG group. Regarding nutritional status, LsTG demonstrated an advantage over both LPG and LTG. Furthermore, remnant gastritis and bile reflux 1 year after surgery was observed more frequently in the LPG group than in the LsTG group. To the best of our knowledge, the present study is the first to compare 
these three types of surgical procedures for proximal gastric carcinoma.

For early gastric cancer located in the upper third of the stomach, there are several surgical options, including reconstruction. Although LTG or LPG is usually performed for early gastric cancer in the upper third of the stomach, short-term outcomes and postoperative quality of life after LTG or LPG are unsatisfactory compared with those after laparoscopic distal gastrectomy (LDG) [5, 6, 22, 23]. LDG has been widely accepted as the standard surgery for cStage I gastric cancer since it was first reported in 1994, and its safety and favorable short-term outcomes have been already established [24-29]. Although LsTG is a technically more complicated and time-consuming procedure than conventional LDG, the incidence of postoperative morbidities was significantly lower after LsTG than that after LTG in the present study. Furthermore, the incidence of anastomotic leakage was a little less after LsTG than that after LPG, although the overall incidence of postoperative morbidities showed no statistical difference. In terms of postoperative morbidities, LsTG may result in favorable outcomes as compared with LTG and LPG.

Previous retrospective studies have documented that distal gastrectomy has advantages over TG in postoperative nutritional status and quality of life [30-32]. On the other hand, it has been reported that PG is better than TG in terms of body weight loss and postoperative symptoms such as diarrhea and dumping syndrome [7, 11, 33]. In the present study, however, nutritional status after LPG was poor or equal to that after LTG. We considered that the relatively poor nutritional status in the LPG group might be a result of older age (median, 70 years) as compared with the LsTG group (median, 65 years) and the LTG group (median, 63.5 years). The present study showed that LsTG had significant advantages in serum albumin and PNI compared with LPG, although patients in the LsTG group tended to have more advanced disease and to receive adjuvant chemotherapy more frequently than those in the LPG group. Furthermore, recovery of postoperative body weight and serum hemoglobin levels was significantly better after LsTG than LTG. We previously proposed that body weight loss after gastrectomy is caused not only by anatomical changes following gastric surgery, but also by a decrease of ghrelin, a gut hormone known to increase appetite [34-38]. The improved nutritional status after LsTG, despite a very small remnant stomach, might result from preservation of the gastric fundus, the primary location of ghrelin secretion [14].

Endoscopic findings at 1 year after surgery showed that significantly fewer patients had gastritis or bile reflux in the LsTG group than in the LPG group, although there was no significant difference in the proportion of patients with reflux esophagitis. Previous studies reported that the incidence of reflux symptoms after PG was significantly higher than that after TG [5, 23]. In our cohort, about $80 \%$ of LPG patients underwent reconstruction by the double-tract method; however, the choice of reconstruction method after PG remains controversial. Although a novel double-flap esophagogastrostomy technique has been recently developed to prevent reflux after PG with esophagogastric anastomosis [39], LsTG could be a good option to prevent reflux esophagitis and remnant gastritis.

In the current version of the Japanese Gastric Cancer Treatment Guidelines, the extent of lymph node dissection differs depending on the type of gastrectomy [16]. Tumors located near the EGJ have a relatively high metastatic rate at lymph node station No. 2. LsTG was not performed in such cases because this procedure does not involve complete dissection of nodes in this station. As for station No. 4sa, it is possible to remove most of the lymph nodes along the short gastric vessels, but complete dissection, including the nodes around the superior pole of the spleen, should be avoided so as to preserve blood supply to the remnant stomach. We therefore consider that LsTG should be performed with complementary procedures such as sentinel node navigation methods for tumors in the greater curvature near the fundus. In addition, the proximal margin could be short in LsTG as compared with LTG or LPG. To ensure the proximal margin for the LsTG patients, we usually put the marking clips approximately $1 \mathrm{~cm}$ proximal to the tumor with confirmation of negative biopsy. The tumor location and marking clips were confirmed by using intraoperative endoscopy before resection, and intraoperative frozen section diagnosis was performed in some cases. Owing to these assistances, all cases in our series revealed negative margin in the final pathological diagnosis.

This study has several limitations. First, it was a retrospective study and therefore a certain degree of selection bias could not be avoided. The surgical procedure in each case was chosen based on a collaborative decision between surgeons and patients as well as on the tumor location. Second, the follow-up period was too short to assess oncological outcomes. Although there was no recurrence in the LsTG group during the follow-up period, the oncological outcomes of LsTG should be assessed in longer-term studies.

Our results suggest that LsTG is a safe procedure compared with LTG and LPG. Furthermore, LsTG may have an advantage over these procedures in terms of postoperative nutritional status and prevention of remnant gastritis. In conclusion, LsTG with a very small remnant stomach could be an alternative treatment option for cStage I proximal gastric carcinoma.

\section{Compliance with ethical standards}

Conflict of interest All authors declare that they have no conflict of interest. 
Ethical standards Written informed consent was obtained from all patients before the procedure. The study was approved by the ethics committee of the Institutional Review Board.

\section{References}

1. Okabayashi T, Gotoda T, Kondo H, Inui T, Ono H, Saito D, et al. Early carcinoma of the gastric cardia in Japan: is it different from that in the West? Cancer (Phila). 2000;89:2555-9.

2. Kunisaki C, Akiyama H, Nomura M, Matsuda G, Otsuka Y, Ono $\mathrm{H}$, et al. Surgical outcomes for early gastric cancer in the upper third of the stomach. J Am Coll Surg. 2005;200:15-9.

3. Takeno S, Hashimoto T, Maki K, Shibata R, Shiwaku H, Yamana I, et al. Gastric cancer arising from the remnant stomach after distal gastrectomy: a review. World J Gastroenterol. 2014;20:13734-40.

4. Hsu CP, Chen CY, Hsieh YH, Hsia JY, Shai SE, Kao CH. Esophageal reflux after total or proximal gastrectomy in patients with adenocarcinoma of the gastric cardia. Am J Gastroenterol. 1997;92:1347-50.

5. An JY, Youn HG, Choi MG, Noh JH, Sohn TS, Kim S. The difficult choice between total and proximal gastrectomy in proximal early gastric cancer. Am J Surg. 2008;196:587-91.

6. Masuzawa T, Takiguchi S, Hirao M, Imamura H, Kimura Y, Fujita $\mathrm{J}$, et al. Comparison of perioperative and long-term outcomes of total and proximal gastrectomy for early gastric cancer: a multiinstitutional retrospective study. World J Surg. 2014;38:1100-6.

7. Takiguchi N, Takahashi M, Ikeda M, Inagawa S, Ueda S, Nobuoka $\mathrm{T}$, et al. Long-term quality-of-life comparison of total gastrectomy and proximal gastrectomy by postgastrectomy syndrome assessment scale (PGSAS-45): a nationwide multi-institutional study. Gastric Cancer. 2015;18:407-16.

8. Yoo CH, Sohn BH, Han WK, Pae WK. Long-term results of proximal and total gastrectomy for adenocarcinoma of the upper third of the stomach. Cancer Res Treat. 2004;36:50-5.

9. Ichikawa D, Komatsu S, Okamoto K, Shiozaki A, Fujiwara H, Otsuji E. Evaluation of symptoms related to reflux esophagitis in patients with esophagogastrostomy after proximal gastrectomy. Langenbecks Arch Surg. 2013;398:697-701.

10. Kinoshita T, Gotohda N, Kato Y, Takahashi S, Konishi M, Kinoshita T. Laparoscopic proximal gastrectomy with jejunal interposition for gastric cancer in the proximal third of the stomach: a retrospective comparison with open surgery. Surg Endosc. 2013;27:146-53

11. Huh YJ, Lee HJ, Oh SY, Lee KG, Yang JY, Ahn HS, et al. Clinical outcome of modified laparoscopy-assisted proximal gastrectomy compared to conventional proximal gastrectomy or total gastrectomy for upper-third early gastric cancer with special references to postoperative reflux esophagitis. J Gastric Cancer. 2015;15:191-200.

12. Jiang X, Hiki N, Nunobe S, Nohara K, Kumagai K, Sano T, et al. Laparoscopy-assisted subtotal gastrectomy with very small remnant stomach: a novel surgical procedure for selected early gastric cancer in the upper stomach. Gastric Cancer. 2011;14:194-9.

13. Kosuga T, Ichikawa D, Komatsu S, Okamoto K, Konishi H, Shiozaki A, et al. Feasibility and nutritional benefits of laparoscopic proximal gastrectomy for early gastric cancer in the upper stomach. Ann Surg Oncol. 2015;22(suppl 3):S929-35.

14. Takiguchi S, Adachi S, Yamamoto K, Morii E, Miyata H, Nakajima K, et al. Mapping analysis of ghrelin producing cells in the human stomach associated with chronic gastritis and early cancers. Dig Dis Sci. 2012;57:1238-46.
15. Japanese Gastric Cancer Association. Japanese classification of gastric carcinoma: 3rd English edition. Gastric Cancer. 2011;14:101-112

16. Japanese Gastric Cancer Association. Japanese gastric cancer treatment guidelines 2014 (ver. 4). Gastric Cancer. 2017;20:1-19

17. Wada N, Kurokawa Y, Takiguchi S, Takahashi T, Yamasaki M, Miyata H, et al. Feasibility of laparoscopy-assisted total gastrectomy in patients with clinical stage I gastric cancer. Gastric Cancer. 2014;17:137-40.

18. Sakuramoto S, Yamashita K, Kikuchi S, Futawatari N, Katada $\mathrm{N}$, Moriya $\mathrm{H}$, et al. Clinical experience of laparoscopy-assisted proximal gastrectomy with Toupet-like partial fundoplication in early gastric cancer for preventing reflux esophagitis. J Am Coll Surg. 2009;209:344-51.

19. Dindo D, Demartines N, Clavien PA. Classification of surgical complications: a new proposal with evaluation in a cohort of 6336 patients and results of a survey. Ann Surg. 2004;240:205-13.

20. Katayama H, Kurokawa Y, Nakamura K, Ito H, Kanemitsu Y, Masuda N, et al. Extended Clavien-Dindo classification of surgical complications: Japan Clinical Oncology Group postoperative complications criteria. Surg Today. 2016;46:668-85.

21. Kubo M, Sasako M, Gotoda T, Ono H, Fujishiro M, Saito D, et al. Endoscopic evaluation of the remnant stomach after gastrectomy: proposal for a new classification. Gastric Cancer. 2002;5:83-9.

22. Lee SE, Ryu KW, Nam BH, Lee JH, Kim YW, Yu JS, et al. Technical feasibility and safety of laparoscopy-assisted total gastrectomy in gastric cancer: a comparative study with laparoscopyassisted distal gastrectomy. J Surg Oncol. 2009;100:392-5.

23. Ahn SH, Lee JH, Park DJ, Kim HH. Comparative study of clinical outcomes between laparoscopy-assisted proximal gastrectomy (LAPG) and laparoscopy-assisted total gastrectomy (LATG) for proximal gastric cancer. Gastric Cancer. 2013;16:282-9.

24. Kitano S, Iso Y, Moriyama M, Sugimachi K. Laparoscopy-assisted Billroth I gastrectomy. Surg Laparosc Endosc. 1994;4:146-8.

25. Kitano S, Shiraishi N, Fujii K, Yasuda K, Inomata M, Adachi Y. A randomized controlled trial comparing open vs laparoscopyassisted distal gastrectomy for the treatment of early gastric cancer: an interim report. Surgery (St. Louis). 2002;131:S306-11.

26. Huscher CG, Mingoli A, Sgarzini G, Sansonetti A, Di Paola M, Recher A, et al. Laparoscopic versus open subtotal gastrectomy for distal gastric cancer: five-year results of a randomized prospective trial. Ann Surg. 2005;241:232-7.

27. Katai H, Mizusawa J, Katayama H, Takagi M, Yoshikawa T, Fukagawa $\mathrm{T}$ et al. Short-term surgical outcomes from a phase III study of laparoscopy-assisted versus open distal gastrectomy with nodal dissection for clinical stage IA/IB gastric cancer: Japan Clinical Oncology Group Study JCOG0912. Gastric Cancer. 2017;20:699-708

28. Yamashita K, Sakuramoto S, Kikuchi S, Futawatari N, Katada N, Hosoda K, et al. Laparoscopic versus open distal gastrectomy for early gastric cancer in Japan: long-term clinical outcomes of a randomized clinical trial. Surg Today. 2016;46:741-9.

29. Colvin H, Mizushima T, Eguchi H, Takiguchi S, Doki Y, Mori M. Gastroenterological surgery in Japan: the past, the present and the future. Ann Gastroenterol Surg. 2017;1:5-10.

30. Nakamura M, Hosoya Y, Yano M, Doki Y, Miyashiro I, Kurashina $\mathrm{K}$, et al. Extent of gastric resection impacts patient quality of life: the Dysfunction After Upper Gastrointestinal Surgery for Cancer (DAUGS32) scoring system. Ann Surg Oncol. 2011;18:314-20.

31. Kobayashi D, Kodera Y, Fujiwara M, Koike M, Nakayama G, Nakao A. Assessment of quality of life after gastrectomy using EORTC QLQ-C30 and STO22. World J Surg. 2011;35:357-64.

32. Kosuga T, Hiki N, Nunobe S, Noma H, Honda M, Tanimura S, et al. Feasibility and nutritional impact of laparoscopy-assisted subtotal gastrectomy for early gastric cancer in the upper stomach. Ann Surg Oncol. 2014;21:2028-35. 
33. Ohashi M, Morita S, Fukagawa T, Oda I, Kushima R, Katai H. Functional advantages of proximal gastrectomy with jejunal interposition over total gastrectomy with Roux-en-Y esophagojejunostomy for early gastric cancer. World J Surg. 2015;39:2726-33.

34. Kojima M, Hosoda H, Date Y, Nakazato M, Matsuo H, Kangawa $\mathrm{K}$. Ghrelin is a growth-hormone-releasing acylated peptide from stomach. Nature (Lond). 1999;402:656-60.

35. Adachi S, Takiguchi S, Okada K, Yamamoto K, Yamasaki M, Miyata $\mathrm{H}$, et al. Effects of ghrelin administration after total gastrectomy: a prospective, randomized, placebo-controlled phase II study. Gastroenterology. 2010;138:1312-20.

36. Yamamoto K, Takiguchi S, Miyata H, Adachi S, Hiura Y, Yamasaki M, et al. Randomized phase II study of clinical effects of ghrelin after esophagectomy with gastric tube reconstruction. Surgery (St. Louis). 2010;148:31-8.
37. Takiguchi S, Murakami K, Yanagimoto Y, Takata A, Miyazaki Y, Mori M, et al. Clinical application of ghrelin in the field of surgery. Surg Today. 2015;45:801-7.

38. Takiguchi S, Miyazaki Y, Takahashi T, Kurokawa Y, Yamasaki M, Nakajima K, et al. Impact of synthetic ghrelin administration for patients with severe body weight reduction more than 1 year after gastrectomy: a phase II clinical trial. Surg Today. 2016;46:379-85.

39. Hayami M, Hiki N, Nunobe S, Mine S, Ohashi M, Kumagai $\mathrm{K}$, et al. Clinical outcomes and evaluation of laparoscopic proximal gastrectomy with double-flap technique for early gastric cancer in the upper third of the stomach. Ann Surg Oncol. 2017;24:1635-42. 